\title{
WEIGHTED COMPOSITION OPERATORS BETWEEN LIPSCHITZ SPACES ON POINTED METRIC SPACES
}

\author{
SAFOURA DANESHMAND AND DAVOOD AlimOHAMMADI
}

Abstract. In this paper, we study weighted composition operators between Banach spaces of scalar-valued Lipschitz functions on pointed metric spaces, not necessarily compact. We give necessary and sufficient conditions for the injectivity and the surjectivity of these operators. We also obtain sufficient and necessary conditions for a weighted composition operator between these spaces to be compact.

Mathematics subject classification (2010): 47B38, 47B33, 46J10.

Keywords and phrases: Compact linear operator, Lipschitz function, pointed metric space, weighted composition operator.

\section{REFERENCES}

[1] F. Botelho And J. Jamison, Composition operators on spaces of Lipschitz functions, Acta Sci. Math. (Szeged) 77, 3-4 (2011), 621-632.

[2] K. ESMAEILI AND H. MAHYAR, Weighted composition operators between vector-valued Lipschitz function spaces, Banach J. Math. Anal. 7, 1 (2013), 59-72.

[3] A. Golbaharan And H. MAHYAR, Weighted composition operators on Lipschitz algebras, Houston J. Math. 42, 3 (2016), 905-917.

[4] A. Jiménez-Vargas and M. Villegas-Vallecillos, Compact composition operators on noncompact Lipschitz spaces, J. Math. Anal. Appl. 398, 1 (2013), 221-229.

[5] H. Kamowitz AND S. ScheinBerg, Some properties of endomorphisms of Lipschitz algebras, Studia Math. 96, 3 (1990), 255-261.

[6] D. R. SHERBERT, Banach algebras of Lipschitz functions, Pacific J. Math. 13 (1963), 1387-1399.

[7] N. WeAVER, Lipschitz, Algebras, World Scientific, Singapore, 1999. 